\title{
Determination of the Axial-Vector Weak Coupling Constant with Ultracold Neutrons
}

J. Liu, ${ }^{1,2}$ M. P. Mendenhall, ${ }^{1}$ A. T. Holley, ${ }^{3}$ H. O. Back, ${ }^{3,4}$ T. J. Bowles, ${ }^{5}$ L. J. Broussard,${ }^{4,6}$ R. Carr, ${ }^{1}$ S. Clayton, ${ }^{5}$ S. Currie, ${ }^{5}$ B. W. Filippone, ${ }^{1}$ A. García, ${ }^{7}$ P. Geltenbort, ${ }^{8}$ K. P. Hickerson, ${ }^{1}$ J. Hoagland, ${ }^{3}$ G. E. Hogan, ${ }^{5}$ B. Hona, ${ }^{9}$ T. M. Ito, ${ }^{5}$ C.-Y. Liu, ${ }^{10}$ M. Makela, ${ }^{5}$ R. R. Mammei, ${ }^{11}$ J. W. Martin, ${ }^{12}$ D. Melconian, ${ }^{13}$ C. L. Morris, ${ }^{5}$ R. W. Pattie, Jr., ${ }^{3,4}$ A. Pérez Galván, ${ }^{1}$ M. L. Pitt, ${ }^{11}$ B. Plaster, ${ }^{9}$ J. C. Ramsey, ${ }^{5}$ R. Rios,${ }^{5,14}$ R. Russell, ${ }^{1}$ A. Saunders, ${ }^{5}$ S. J. Seestrom, ${ }^{5}$ W. E. Sondheim, ${ }^{5}$ E. Tatar, ${ }^{13}$ R. B. Vogelaar, ${ }^{11}$ B. VornDick, ${ }^{3}$ C. Wrede,${ }^{7}$ H. Yan, ${ }^{9}$ and A. R. Young ${ }^{3,4}$

(UCNA Collaboration)

${ }^{1}$ Kellogg Radiation Laboratory, California Institute of Technology, Pasadena, California 91125, USA

${ }^{2}$ Department of Physics, Shanghai Jiao Tong University, Shanghai, 200240, China

${ }^{3}$ Department of Physics, North Carolina State University, Raleigh, North Carolina 27695, USA

${ }^{4}$ Triangle Universities Nuclear Laboratory, Durham, North Carolina 27708, USA

${ }^{5}$ Los Alamos National Laboratory, Los Alamos, New Mexico 87545, USA

${ }^{6}$ Department of Physics, Duke University, Durham, North Carolina 27708, USA

${ }^{7}$ Department of Physics, University of Washington, Seattle, Washington 98195, USA

${ }^{8}$ Institut Laue-Langevin, 38042 Grenoble Cedex 9, France

${ }^{9}$ Department of Physics and Astronomy, University of Kentucky, Lexington, Kentucky 40506, USA

${ }^{10}$ Department of Physics, Indiana University, Bloomington, Indiana 47408, USA

${ }^{11}$ Department of Physics, Virginia Tech, Blacksburg, Virginia 24061, USA

${ }^{12}$ Department of Physics, University of Winnipeg, Winnipeg, MB R3B 2E9, Canada

${ }^{13}$ Cyclotron Institute, Texas AM University, College Station, Texas 77843, USA

${ }^{14}$ Department of Physics, Idaho State University, Pocatello, Idaho 83209, USA

(Received 21 July 2010; published 29 October 2010; corrected 9 November 2010)

\begin{abstract}
A precise measurement of the neutron decay $\beta$ asymmetry $A_{0}$ has been carried out using polarized ultracold neutrons from the pulsed spallation ultracold neutron source at the Los Alamos Neutron Science Center. Combining data obtained in 2008 and 2009, we report $A_{0}=-0.11966 \pm 0.00089_{-0.001}^{+0.001} 23$, from which we determine the ratio of the axial-vector to vector weak coupling of the nucleon $g_{A} / g_{V}=$ $-1.27590_{-0.004}^{+0.004}$.
\end{abstract}

DOI: $10.1103 /$ PhysRevLett.105.181803

PACS numbers: 13.30.Ce, 12.15.Hh, 14.20.Dh, 23.40.Bw

The axial-vector weak coupling constant $g_{A}$ plays an important role in our understanding of the nucleon spin and flavor structure [1,2]. It is a central target for high precision lattice QCD calculations [3,4] and an essential parameter in effective field theories [5]. $g_{A}$ is also important in a variety of astrophysical processes, including solar fusion reaction rates [6].

The angular distribution of emitted electrons from polarized neutron decay can be expressed as $W(E) \propto 1+\frac{v}{c} \times$ $\langle P\rangle A(E) \cos \theta$, where $A(E)$ specifies the $\beta$ asymmetry versus electron energy $E, v$ is the electron velocity, $c$ is the speed of light, $\langle P\rangle$ is the mean polarization, and $\theta$ is the angle between the neutron spin and the electron emission direction [7]. The leading order value of $A(E), A_{0}$, is given by

$$
A_{0}=\frac{-2\left(\lambda^{2}-|\lambda|\right)}{1+3 \lambda^{2}}
$$

where $\lambda=g_{A} / g_{V}$ and $g_{V}$ is the vector weak coupling constant with $g_{V}=1$ under the conserved vector current (CVC) hypothesis of the standard model [8]. Higher order terms in $A(E)$ are at the $1 \%$ level, and can be calculated precisely under the standard model $[9,10] . g_{A}$ can also be indirectly determined by combining the Fermi coupling constant $G_{F}$ (measured to 5 ppm using muon decay [11]), the Cabibbo-Kobayashi-Maskawa (CKM) quark mixing matrix element $\left|V_{\text {ud }}\right|$ (measured to $225 \mathrm{ppm}$ using $0^{+} \rightarrow$ $0^{+}$superallowed decays [8]), and the neutron lifetime (measured to $0.9 \%[12,13]$ ). Thus, a measurement of the $\beta$ asymmetry permits the direct determination of $g_{A}$, as well as a robust test of the consistency of measured neutron $\beta$-decay observables under the standard model.

In order to obtain $A(E)$, one must determine the polarization of the neutron beam and control all sources of systematic uncertainty due to backgrounds, including those produced by the neutrons themselves, the detector response, and electronevent reconstruction. All previous precise measurements of the $\beta$ asymmetry [14-17] have been performed with coldneutron beams and have shown a range of results much wider than the reported uncertainties [12]. Our measurement, UCNA, utilizes ultracold neutrons (UCN-neutrons with kinetic energy less than $200 \mathrm{neV}$ ) and controls key systematic uncertainties: neutron polarization and neutron-generated 
backgrounds. In 2007, we carried out a proof-of-principle $\beta$-asymmetry measurement [18]. At present, the UCNA experiment is characterized by neutron polarizations greater than $99.48 \%$ and neutron-generated backgrounds that produce corrections to the asymmetry below the $0.02 \%$ level.

Some of the experimental details of UCNA are explained in [18]. We used the UCN source at the Los Alamos Neutron Science Center accelerator at Los Alamos National Laboratory [19]. The UCN were polarized by a $7 \mathrm{~T}$ primary polarizer coupled to an adiabatic fast passage spin flipper to control the spin state $[18,20]$. Polarized UCN entered the superconducting spectrometer [21] and were confined in a $3 \mathrm{~m}$ long, $12.4 \mathrm{~cm}$ diameter electropolished $\mathrm{Cu}$ tube (decay trap) with variable thickness mylar end caps. The inside surface of each end cap was coated with $200 \mathrm{~nm}$ of Be. A $1 \mathrm{~T}$ magnetic field was oriented parallel to the decay trap, along which decay electrons spiraled toward one of two identical electron detector packages, each covering a $2 \pi$ sr angular hemisphere. Each detector package consisted of a lowpressure multiwire proportional chamber (MWPC) [22] backed by a plastic scintillator, with scintillation light measured by four photomultiplier tubes (PMT). Each MWPC had thin front and back mylar windows which separated low- $Z$ chamber gas (neopentane) from the spectrometer vacuum. To study key systematics due to electron energy loss and backscattering in the windows, we operated the experiment in four different geometries with different decay trap end caps and MWPC window thicknesses-A: 0.7 and 25; B: 13.2 and 25; C: 0.7 and 6; and D [23]: 0.7 and $6 \mu \mathrm{m}$, respectively.

Cosmic-ray muon backgrounds were identified by a combination of plastic scintillator veto paddles and sealed drift tube assemblies [24] surrounding the electron detectors. $\gamma$-ray backgrounds were vetoed by a coincidence between the MWPC and the main $\beta$ scintillator.

A gate valve separated the UCN source from the experimental apparatus. A typical run unit consisted of a background run (gate valve closed), a $\beta$-decay run (gate valve open), and a UCN depolarization run to measure the equilibrium UCN polarization for the accompanying $\beta$-decay run. The UCN spins were flipped back and forth (while the magnetic field in the superconducting spectrometer was held fixed) between run units [Fig. 1(a)], which partially canceled systematic rate variation over the period of a spin cycle $(\sim 1.5 \mathrm{~h})$. During a depolarization run, the guide serving as input to the $7 \mathrm{~T}$ polarizing field was first connected to a UCN detector [25] so that UCN exiting the experiment could be counted, while the gate valve was closed and the proton pulses [19] were discontinued. This cleaning phase, which lasted $25 \mathrm{~s}$, produced a signal in the $\mathrm{UCN}$ detector proportional to the number of correctly polarized UCN present in the experimental geometry at the end of the $\beta$-decay measurement interval. Following the cleaning phase, the state of the spin flipper was changed, allowing only incorrectly polarized UCN

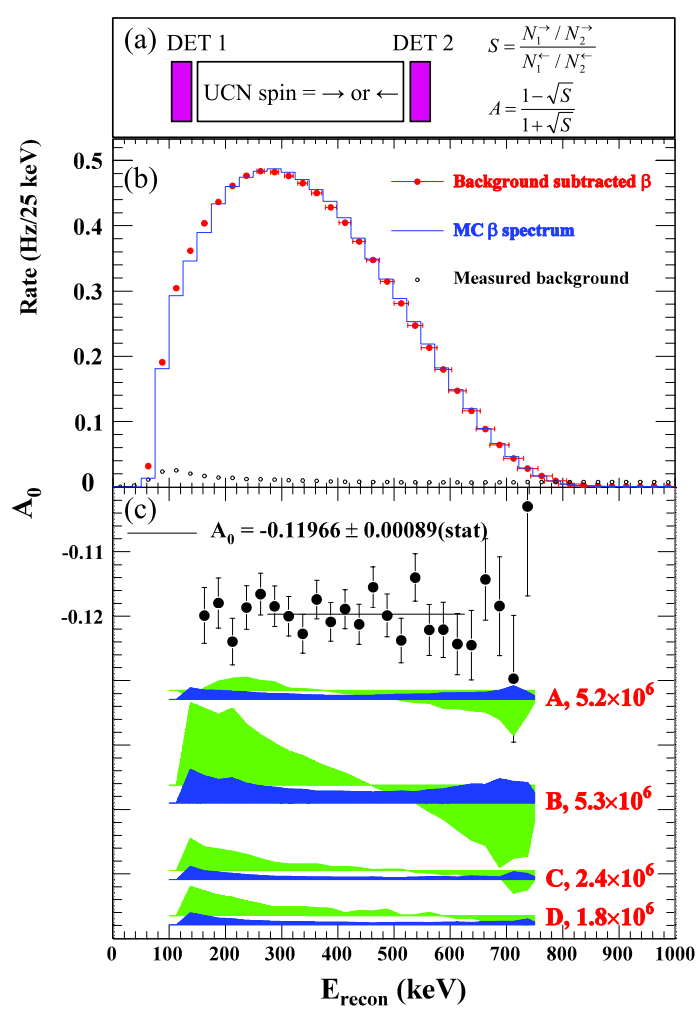

FIG. 1 (color online). Panel (a): schematic of the experiment and definition of the asymmetry. Panel (b): background subtracted electron $E_{\text {recon }}$ spectrum (solid circles with the uncertainty of $E_{\text {recon }}$ reflected by the horizontal error bars), combining both sides of the detectors and two spin states, overlaid with the Monte Carlo spectrum (histogram). The open circles represent the measured ambient background spectrum. Panel (c): $A_{0}$ vs

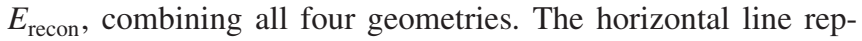
resents the extracted $A_{0}$ within $[275,625] \mathrm{keV}$. Drawn on the same scale below the graph are four sets of bands representing the sum of energy-dependent backscattering and angle effect corrections (light color), the positive sign indicating a larger $\left|A_{0}\right|$, and their uncertainties (dark) for the four geometries. The positive (negative) correction at low (high) energy is a consequence of the backscattering (angle effect) dominating in this energy region. The number of $\beta$ events for each geometry is also indicated in the figure.

remaining downstream of the spin flipper to pass through the $7 \mathrm{~T}$ polarizing field and be counted. Counting during this unloading phase was performed for $\sim 200 \mathrm{~s}$ in order to measure background as well as incorrectly polarized UCNs. Since the measured depolarization was consistent with zero at the $1 \sigma$ level, we folded together statistical and systematic errors to produce a global polarization lower limit of $99.48 \%$ at the $68 \%$ CL [20], covering all four geometries and both polarization states.

The experimental triggers were formed by requiring at least two of the four PMT signals over threshold in either of the scintillator detectors. Electron positions were determined with the MWPC to an accuracy of better than $2 \mathrm{~mm}$ based on the distribution of charge on two 
perpendicular cathode grids in the MWPC [22]. A fiducial cut of $r<45 \mathrm{~mm}$ was placed on the trigger side to reduce background and to eliminate electrons that could strike the decay trap walls.

Reconstructed event energies $E_{\text {recon }}$ were measured using the signals from the scintillator PMTs as calibrated with conversion electron sources $\left({ }^{109} \mathrm{Cd},{ }^{139} \mathrm{Ce},{ }^{113} \mathrm{Sn},{ }^{85} \mathrm{Sr}\right.$, and ${ }^{207} \mathrm{Bi}$ ). The position dependence of the response of each PMT was mapped out by comparing the neutron betadecay spectrum endpoint observed at different positions. The energy reconstruction uncertainty was determined to be the larger of $\pm 5 \mathrm{keV}$ or $\pm 2.5 \%$, which covered the uncertainty in the position response of the scintillator, as well as possible variation of the energy response allowed by the calibration data.

The PMT gains were monitored based on frequent calibrations with a removable ${ }^{113} \mathrm{Sn}$ calibration source, which also measured the energy resolution of the system $(\sim 400$ photoelectrons per $\mathrm{MeV}$ ), and by observing shifts in the minimum-ionizing peak of cosmic-ray muons during $\beta$-decay-background runs.

The majority of the $\beta$-decay events were single detector triggers. However, due to electron backscattering, a small fraction of the events, varying between $1.7 \%$ and $3.4 \%$ for the four geometries, triggered both scintillators, and another small fraction $(\sim 2 \%)$ were detected by both MWPCs, but triggered only one of the scintillators. In the first case, the initial direction of the electron could be determined by the relative timing of the triggers, while in the second case a fixed cut $(4.1 \mathrm{keV})$ or a likelihood function based on the energy loss in the trigger side MWPC yielded an identification efficiency of $\sim 80 \%$ based on Monte Carlo calculations (discussed later).

In addition to the ambient backgrounds (measured with the UCN gate valve closed and suppressed by the pulsed nature of the UCN source [19]), which were subtracted run by run, neutron captures in the vicinity of the detectors could create prompt $\gamma$ 's with energies up to $\sim 8 \mathrm{MeV}$, generating an irreducible background in the experiment. This background was significantly suppressed, compared to cold-neutron beam experiments, by the relatively low density and low capture and upscatter probability of neutrons in and around the spectrometer. Combining direct measurements with Monte Carlo calculations, we obtained an upper limit of $0.02 \%$ on the correction to the asymmetry.

For each run, events were sorted into $25 \mathrm{keV} E_{\text {recon }}$ bins from 0 to $1200 \mathrm{keV}$ and assigned an initial direction. The rates in the two detectors were then computed based on the experiment live time. We applied separate spin-dependent blinding factors to the two detector rates, effectively adding an unknown scaling factor to the measured asymmetry that was constrained to be within $1.00 \pm 0.05$. After determination of all cuts, corrections, and uncertainties, this factor was removed. For each $\beta$-decay-background run pair, the background rate was subtracted from the $\beta$-decay-run rate bin by bin. The reconstructed energy spectrum (background subtracted, averaged over the two spin states) is shown in panel (b) of Fig. 1, overlaid with the measured background. The $S / B$ is about 40 in our analysis energy window between 275 and $625 \mathrm{keV}$ (discussed later). Also overlaid is the Monte Carlo-predicted reconstructed energy spectrum, with all detector effects (efficiencies, resolutions, etc.) taken into account. The systematic effect due to the small discrepancy between the two spectra is well covered by the energy reconstruction uncertainty in Table I.

For a given geometry, a "super ratio" of count rates among the two detectors and UCN spin states was calculated (as defined in [18]), from which the raw measured asymmetry was determined [see also Fig. 1(a)]. To extract $A_{0}$, we first multiplied the raw measured asymmetry by $1 /\langle v / c\rangle$ in each energy bin to remove the strongest energy dependence. As in [18], two scattering-related effects dominated subsequent systematic corrections: the residual backscattering correction and the angle effect. In addition to a small residual correction due to incorrect identification of the initial electron direction for the measured electron backscatters (where both detectors observed the electron), there were corrections for backscattering from the decay trap windows and the front windows of the MWPC that could not be identified experimentally. Angle effects arose from the fact that the energy loss of an electron in the thin windows was strongly angle dependent. Low-energy, large

TABLE I. Summary of experimental corrections and uncertainties in \% (all fractional to $A_{0}$ ). Upper table: geometryindependent effects (with zero corrections). Lower table: geometry-dependent effects (first value $=$ correction, second value $=$ uncertainty in each column), with $\sigma_{\text {stat }}, \Delta_{\text {back }}$, $\Delta_{\text {ang }}$, and $\epsilon_{\text {MWPC }}$ referring to statistical uncertainty, backscattering correction, angle effect correction, and the uncertainty associated with MWPC inefficiency, respectively.

\begin{tabular}{|c|c|c|c|c|c|c|c|c|}
\hline \multicolumn{7}{|c|}{ Geometry-independent effect } & \multicolumn{2}{|c|}{ Uncertainty $(\%)$} \\
\hline \multicolumn{7}{|c|}{ Polarization } & \multicolumn{2}{|c|}{+0.52} \\
\hline \multicolumn{7}{|c|}{ Field nonuniformity } & \multicolumn{2}{|c|}{+0.20} \\
\hline \multicolumn{7}{|c|}{ Rate-dependent gain shift } & \multicolumn{2}{|c|}{0.08} \\
\hline \multicolumn{7}{|c|}{ Gain fluctuation } & \multicolumn{2}{|c|}{0.20} \\
\hline \multicolumn{7}{|c|}{ Dead time } & \multicolumn{2}{|c|}{0.01} \\
\hline \multicolumn{7}{|c|}{ Energy reconstruction } & \multicolumn{2}{|c|}{0.47} \\
\hline \multicolumn{7}{|c|}{ UCN-induced background } & \multicolumn{2}{|c|}{0.02} \\
\hline \multicolumn{7}{|c|}{ Muon veto efficiency } & \multicolumn{2}{|c|}{0.30} \\
\hline \multicolumn{7}{|c|}{ Live time uncertainty } & \multicolumn{2}{|c|}{0.24} \\
\hline \multicolumn{7}{|c|}{ Fiducial cut } & \multicolumn{2}{|c|}{0.24} \\
\hline \multicolumn{9}{|c|}{ Geometry-dependent effect } \\
\hline \multicolumn{3}{|c|}{$\mathrm{A}(\%)$} & \multicolumn{2}{|c|}{$\mathrm{B}(\%)$} & \multicolumn{2}{|c|}{$\mathrm{C}(\%)$} & \multicolumn{2}{|c|}{$\mathrm{D}(\%)$} \\
\hline$\sigma_{\text {stat }}$ & $\cdots$ & 1.23 & $\cdots$ & 1.22 & $\cdots$ & 2.00 & $\cdots$ & 2.10 \\
\hline$\Delta_{\text {back }}$ & 1.34 & \pm 0.40 & 4.32 & \pm 1.30 & 1.07 & \pm 0.32 & 1.08 & \pm 0.32 \\
\hline$\Delta_{\text {ang }}$ & -1.81 & \pm 0.45 & -3.22 & \pm 0.81 & -0.60 & \pm 0.15 & -0.36 & \pm 0.09 \\
\hline$\epsilon_{\mathrm{MWPC}}$ & 0 & 0.02 & 0 & 0.01 & 0 & 0.16 & 0 & 0.5 \\
\hline
\end{tabular}




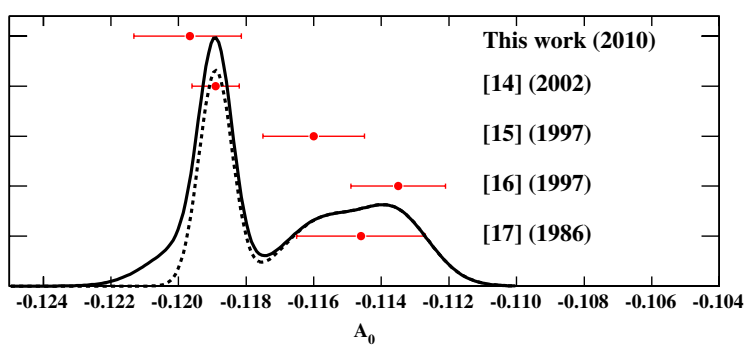

FIG. 2 (color online). Ideogram comparing the previous measurements that are included in the 2009 Particle Data Group best value for $A_{0}$ along with the new result reported here. The dashed line is the ideogram with the previous four data points, and the solid line shows the result of including the present data.

pitch angle electrons were more likely to fall below the scintillator threshold, leading to a suppression of the acceptance at large angles $(\langle\cos \theta\rangle$ deviating from $1 / 2)$. Both of these effects were evaluated with two independent simulation programs: Penelope [26] and GEANT4 [27]. The resulting corrections for all four geometries are shown in Table I. Based on the observed difference between the calculations and the data, we assigned an uncertainty of $30 \%$ to the backscattering correction and $25 \%$ to the angle effect correction.

Recoil-order corrections to $A(E)$ (see also [18]) were calculated within the context of the standard model according to the formalism of $[9,10]$, leading to a correction of $(-1.79 \pm 0.03) \%$ to $A_{0}$. The value for the radiative correction to $A_{0}$ was taken from the calculations of [28], yielding a small theoretical correction of $(0.10 \pm 0.05) \%$.

Applying all corrections mentioned above, the extracted $A_{0}$ is plotted against $E_{\text {recon }}$ (all geometries combined) in panel (c) of Fig. 1. Energy-dependent corrections (backscattering and angle effects) and their uncertainty are indicated as bands in the figures. The final $A_{0}$ is obtained from a constant fit over a range of energy [29]. The energy window, 275 to $625 \mathrm{keV}$, was chosen to optimize combined statistical and systematic uncertainties before unblinding the asymmetries. The value of $A_{0}$ was insensitive to the choice of energy window, with the variation less than $15 \%$ of the statistical uncertainty for windows between 150 and $750 \mathrm{keV}$.

The experimental uncertainties and systematic corrections to $A_{0}$ are summarized in Table I. Geometry-dependent systematic uncertainties (backscattering, angle effect, and MWPC inefficiency) are treated as completely correlated among the different geometries. Combining the four geometries, we find $A_{0}=-0.11966 \pm 0.00089_{-0.00140}^{+0.001 ~} 23$ (with a $\chi^{2} / \nu$ of 2.4/3), where the first uncertainty is statistical and the second systematic [29]. Based on Eq. (1), we then determine $g_{A} / g_{V}=-1.27590_{-0.00445}^{+0.004}=g_{A}$, where the second equality assumes CVC [8].

Our result for $A_{0}$ is compared with the world data [14-17] in Fig. 2. Our result is in good agreement with the most recent and precise result for $A_{0}$ [14]. We note that the direct extraction of $g_{A} / g_{V}$ from the $\beta$ asymmetry is, unlike extraction from the neutron lifetime [12,30], independent of the Cabibbo-Kobayashi-Maskawa matrix element $\left|V_{\text {ud }}\right|$. This strongly motivates UCN-based measurements of the neutron $\beta$ asymmetry, where the key neutron-related systematic uncertainties can be reduced below the $0.1 \%$ level. Recently demonstrated improvements to the UCN source and refinement of the energy response and gain monitoring will permit the collection of a much larger data set and the reduction of all major systematic uncertainties.

This work was supported in part by the Department of Energy Office of Nuclear Physics, National Science Foundation (NSF-0555674, NSF-0855538, NSF0653222), and the Los Alamos National Laboratory LDRD program. We gratefully acknowledge the support of the LANSCE and AOT divisions of Los Alamos National Lab.

[1] S. D. Bass, Rev. Mod. Phys. 77, 1257 (2005).

[2] F. E. Close and R. G. Roberts, Phys. Rev. Lett. 60, 1471 (1988).

[3] T. Yamazaki et al. (RBC+UKQCD Collaborations), Phys. Rev. Lett. 100, 171602 (2008).

[4] K.-S. Choi, W. Plessas, and R. F. Wagenbrunn, Phys. Rev. C 81, 028201 (2010).

[5] M. Göckeler et al. (QCDSF Collaboration), Phys. Rev. D 71, 034508 (2005).

[6] E. G. Adelberger et al., arXiv:1004.2318.

[7] J. D. Jackson, S. B. Treiman, and H. W. Wyld, Jr., Phys. Rev. 106, 517 (1957).

[8] I. S. Towner and J. C. Hardy, Rep. Prog. Phys. 73, 046301 (2010).

[9] D. H. Wilkinson, Nucl. Phys. A377, 474 (1982).

[10] S. Gardner and C. Zhang, Phys. Rev. Lett. 86, 5666 (2001).

[11] D. B. Chitwood et al., Phys. Rev. Lett. 99, 032001 (2007).

[12] C. Amsler et al. (Particle Data Group), Phys. Lett. B 667, 1 (2008) and 2009 partial update for the 2010 edition.

[13] A. Czarnecki, W. J. Marciano, and A. Sirlin, Phys. Rev. D 70, 093006 (2004).

[14] H. Abele et al., Phys. Rev. Lett. 88, 211801 (2002).

[15] P. Liaud et al., Nucl. Phys. A612, 53 (1997).

[16] B. G. Yerozolimsky et al., Phys. Lett. B 412, 240 (1997).

[17] P. Bopp et al., Phys. Rev. Lett. 56, 919 (1986).

[18] R. W. Pattie et al., Phys. Rev. Lett. 102, 012301 (2009).

[19] A. Saunders et al., Phys. Lett. B 593, 55 (2004); M. Makela et al. (to be published).

[20] A. T. Holley et al. (to be published).

[21] B. Plaster et al., Nucl. Instrum. Methods Phys. Res., Sect. A 595, 587 (2008).

[22] T. M. Ito et al., Nucl. Instrum. Methods Phys. Res., Sect. A 571, 676 (2007).

[23] The entrance guide for Geometry D was diamondlikecarbon-coated $\mathrm{Cu}$, as compared to bare $\mathrm{Cu}$ for the other 
geometries, resulting in a different UCN velocity spectrum in the decay trap.

[24] R. Rios et al., Nucl. Instrum. Methods Phys. Res., Sect. A (to be published).

[25] C.L. Morris et al., Nucl. Instrum. Methods Phys. Res., Sect. A 599, 248 (2009).

[26] J. Sempau et al., Nucl. Instrum. Methods Phys. Res., Sect. B 132, 377 (1997).
[27] S. Agostinelli et al., Nucl. Instrum. Methods Phys. Res., Sect. A 506, 250 (2003); http://geant4.cern.ch/.

[28] F. Glück and K. Toth, Phys. Rev. D 46, 2090 (1992).

[29] D. Stump et al., Phys. Rev. D 65, 014012 (2001).

[30] The unresolved discrepancy between the most recent lifetime measurement and the previous ones is discussed in the summary of neutron properties in [12]. 